\title{
THE NAVY PRECISION OPTICAL INTERFEROMETER (NPOI): AN UPDATE
}

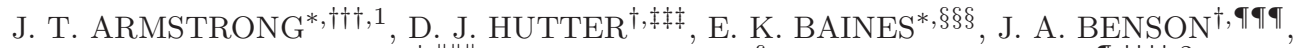 \\ R. M. BEVILACQUA ${ }^{\ddagger},\|\| \|$, T. BUSCHMANN ${ }^{\S, * * * *}$, J. H. CLARK III $\boldsymbol{\Phi}^{\mathbb{1}, \dagger \dagger \dagger, 2}$,$$
\text { A. GHASEMPOUR }{ }^{\|, \ddagger \ddagger \ddagger}, \text { J. C. HALL }{ }^{* *}, \S \S \S \S, \text { R. B. HINDSLEY*, ๆ }
$$

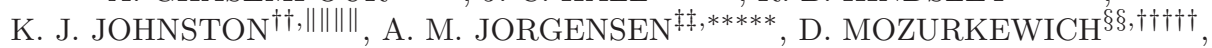$$
\text { M. W. MUTERSPAUGH }
$$

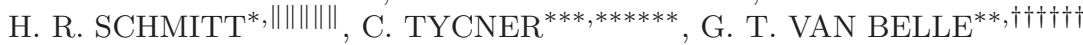$$
\text { and R. T. ZAVALA }{ }^{\dagger}
$$ \\ * Remote Sensing Division, Code 7215 \\ US Naval Research Laboratory, 4555 Overlook Ave. SW \\ Washington, DC 20375, USA \\ ${ }^{\dagger}$ US Naval Observatory Flagstaff Station \\ 10391 W. Naval Observatory Rd. \\ Flagstaff, AZ 86001, USA \\ ${ }^{\ddagger}$ Remote Sensing Division, Code 7200, USA \\ US Naval Research Laboratory, 4555 Overlook Ave. NW \\ Washington, DC 20375 \\ $\S$ Arizona Embedded Systems LLC, 677 N. Fox Hill Rd. \\ Flagstaff, AZ 86004, USA \\ ฯ Remote Sensing Division, Code 7216 \\ US Naval Research Laboratory \\ "Optical Spectroscopy Division \\ HORIBA Scientific, 3880 Park Ave. \\ Edison, NJ 08820, USA \\ ** Lowell Observatory, 1400 W. Mars Hill Rd. \\ Flagstaff, AZ 86001, USA \\ ${ }^{\dagger} 7305$ Wickford Dr., Alexandria, VA 22315, USA \\ $\ddagger_{\ddagger}$ Electrical Engineering Department \\ New Mexico Institute of Mining and Technology, 801 Leroy Place \\ Socorro, NM 87801, USA \\ ${ }^{\S}$ Seabrook Engineering, 9310 Dubarry Rd. \\ Seabrook, MD 20706, USA \\ ฯ Center of Excellence in Information Systems and \\ Department of Mathematical Sciences \\ College of Engineering, Tennessee State University \\ 3500 John A. Merritt Blvd. \\ Box No. 9501, Nashville, TN 37209, USA
}

\footnotetext{
${ }^{1}$ Corresponding author.

${ }^{2}$ Mailing address: US Naval Observatory Flagstaff Station, 10391 W. Naval Observatory Rd., Flagstaff, AZ 86001, USA.
} 


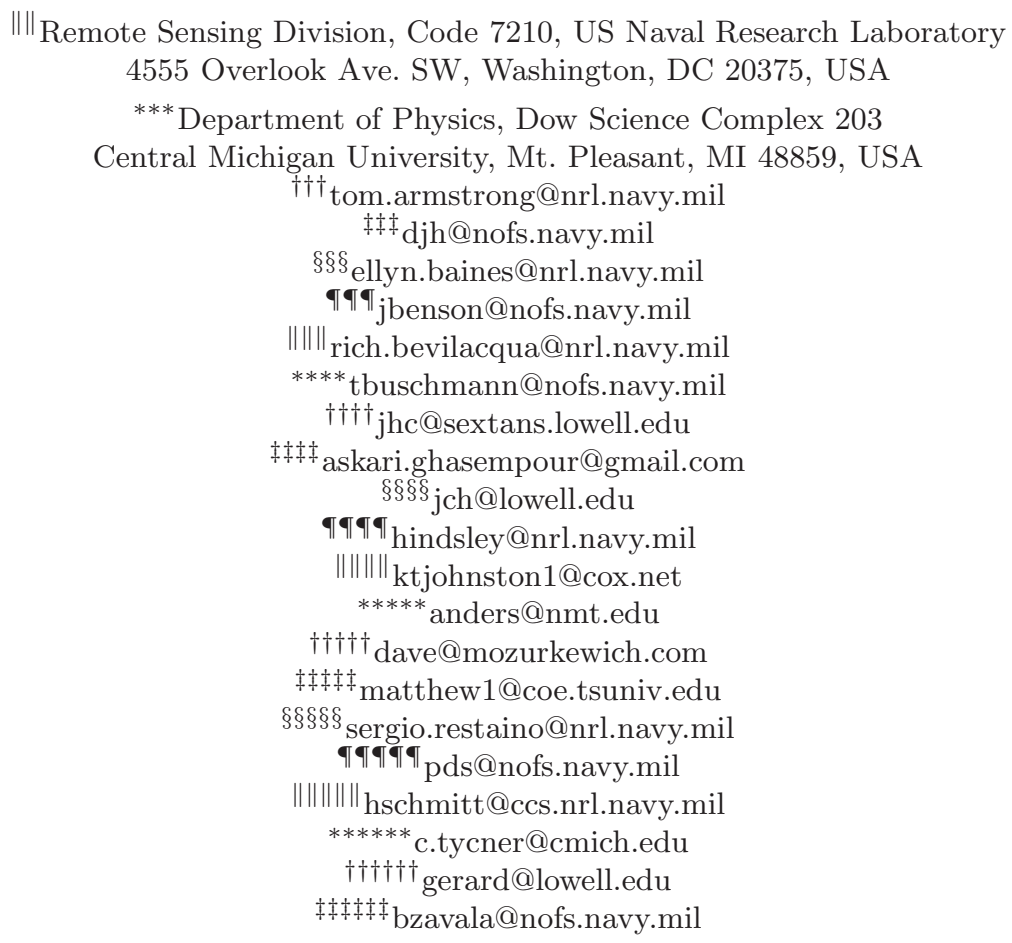

Received 2013 June 29; Accepted 2013 August 24; Published 2014 January 6

\begin{abstract}
The Navy Precision Optical Interferometer (NPOI) has two purposes: wide angle precise astrometry and high-resolution imaging, both at visible wavelengths. It operates with up to six 12-cm diameter apertures simultaneously, with baseline lengths (distances between array elements) from $16 \mathrm{~m}$ to $79 \mathrm{~m}$, and disperses the combined light into 16 spectral channels. It has been operational since first fringes were found in 1994; six-beam operations began in 2001. The NPOI is undergoing upgrades in numerous areas: control system and data acquisition improvements, a second beam combiner, additional array stations for both longer and shorter baselines, and supplemental delay lines to improve sky coverage for the longer baselines. Future possibilities include the installation of four fixed $1.8 \mathrm{~m}$ telescopes as well as up to six moveable lightweight $1.4 \mathrm{~m}$ telescopes. Observing programs, including programs led by outside investigators, cover a broad range of stellar astrophysics as well as observations of geostationary satellites.
\end{abstract}

Keywords: Instrumentation: interferometers; techniques: interferometric.

\section{Introduction}

In October, 1994, when the Navy Precision Optical Interferometer (NPOI), then known as the Navy Prototype Optical Interferometer, produced its first fringes on a stellar target, six other optical and infrared interferometers were in operation: I2T, GI2T, ISI, SUSI, COAST, and IOTA. ${ }^{(a)}$ Since that time, four of these have closed: I2T, COAST, GI2T, and IOTA. Three others have opened and closed: PTI, MIRA-I.2, ${ }^{(b)}$ and the Keck Interferometer. ISI, SUSI, and NPOI are still operating. In addition, three major interferometers have opened: the CHARA Array, VLTI, and the LBT Interferometer. ${ }^{(c)}$

\footnotetext{
${ }^{a}$ Respectively, these are the Interféromètre à 2 Télescopes, Grand Interféromètre à 2 Télescopes, Infrared Spatial Interferometer, Sydney University Stellar Interferometer, Cambridge Optical Aperture Synthesis Telescope, and Infrared Optical Telescope Array. Proc. SPIE 2200 (1994) has papers on each.
}

\footnotetext{
${ }^{\mathrm{b}}$ Palomar Testbed Interferometer and Mitaka opticalInfraRed Array.

${ }^{\mathrm{c}}$ Center for High Angular Resolution Astronomy, the Very Large Telescope Interferometer, and the Large Binocular Telescope.
} 
As optical/infrared interferometery has developed, the NPOI has grown in the number of array elements and maximum baseline length - more slowly than we wished but at a quickened place in the last three years. The last general introduction to the NPOI appeared in 1998 (Armstrong et al., 1998b), four years after first fringes, but three years before full six-element observations were first undertaken. Updates describing subsequent developments have appeared in Mozurkewich et al. (2003), Benson et al. (2004), Johnston et al. (2006), and Hutter et al. (2008). In this paper, we will describe the current state of the NPOI as well as our expectations for the near future and ambitions beyond that. Along the way, we will briefly mention some of the observational programs undertaken with the NPOI.

\section{A Brief Description}

The NPOI operates at visual wavelengths using as many as six array elements simultaneously. It is a dual purpose instrument, intended for both wideangle astrometry and imaging. The array elements are siderostats that feed a $12 \mathrm{~cm}$ diameter vacuum beam transport system conveying light to the optics laboratory, where access to the pathlength compensation systems is provided and where the two beam combiners are housed.

Three institutions are involved in the construction and operation of the NPOI. Although there is considerable overlap in roles, the US Naval Observatory oversees operations and has a primary interest in the astrometric program; the US Naval Research Laboratory provides engineering support and has a primary interest in interferometric imaging; and the Lowell Observatory provides infrastructure support and observing staff under contract with the Navy, and is an active observing partner.

\subsection{Array layout}

The NPOI consists of an astrometric sub-array of four siderostats and an imaging sub-array of six siderostats. The astrometric array is a $\mathrm{Y}$ with baselines from $19 \mathrm{~m}$ to $38 \mathrm{~m}$. The astrometric siderostats, which are fixed in position, are equipped with laser metrology to monitor their motions (Hutter \& Elias, 2003). The astrometric system also includes a laser "constant term" metrology system to monitor changes in the feed system from the beam combiner to the astrometric siderostats (Benson et al., 2004).
The imaging array is laid out in a $\mathrm{Y}$ with $250 \mathrm{~m}$ arms, with a total of 30 stations where siderostats can be placed. The imaging siderostats are designed to be moveable and are not equipped with metrology. The two siderostat types are used together for imaging observations. Possible baseline lengths range from $2.2 \mathrm{~m}$ to $437 \mathrm{~m}$. The station locations along the arms were chosen to allow equal spacing between siderostats in order to optimize baseline bootstrapping for imaging stellar surfaces (see Armstrong et al., 1998b), but unequal spacing is of course also available. The current configuration is shown in Fig. 1.

Each siderostat feeds a $20 \mathrm{~cm}$ tip-tilt mirror that in turn sends the starlight through a window into the vacuum feed system shared by both the astrometric and the imaging arrays. The feed beam mirrors limit the available aperture of the siderostats to $12 \mathrm{~cm}$ despite the fact that the siderostats are $50 \mathrm{~cm}$ in diameter, a legacy of earlier plans for beam-compressing telescopes.

\subsection{Delay systems}

In the laboratory, periscopes lower the incoming beams to a common horizontal plane and send them on to the "fast delay lines" (FDLs), which provide up to $35 \mathrm{~m}$ of continuously-variable delay for each beam line. In addition to providing pathlength variation to counteract earth rotation, they also compensate for effective pathlength variations due to atmospheric turbulence, and they impose

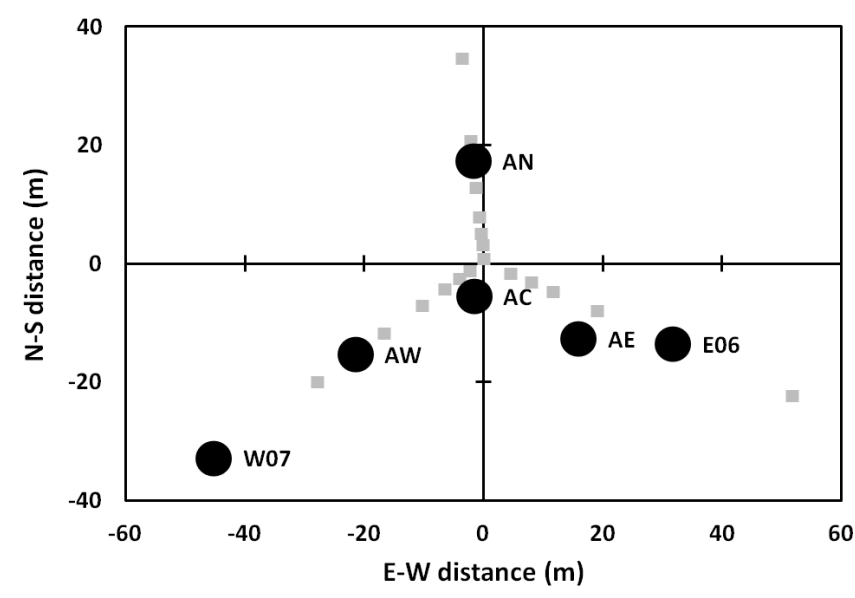

Fig. 1. The current NPOI configuration. The available stations (filled circles) include two imaging stations (W07, E06) and the four astrometric stations. The small gray squares are station locations not yet in use. The three arms of the imaging array $\mathrm{Y}$ extend beyond the borders of the figure. 
triangle-wave delay modulation when the Classic combiner is in use.

For baselines longer than $\sim 80 \mathrm{~m}$, additional delay is needed to allow full sky coverage. The "long delay lines" (LDLs), described by Armstrong et al. (1998b), are designed to provide up to $\sim 520 \mathrm{~m}$ of additional delay in increments of $29 \mathrm{~m}$ with appropriately-spaced pop-up mirrors along their $\sim 130 \mathrm{~m}$ length (light traverses the LDLs four times). When the LDLs are operational, foreseen for 2014, the periscopes will divert the light beams to the LDLs, which extend northward from the laboratory, before sending them on to the FDLs. Currently, however, incoming light bypasses the LDLs.

\subsection{Beam combiners}

Two beam combiners are available. The first, the "Classic" combiner (Mozurkewich, 1994), uses avalanche photo-diodes (APDs) as detectors. It depends on triangle-wave delay line modulation, sampling the combined light in 64 bins along each half of the $2 \mathrm{~ms}$ stroke to detect fringes temporally, and provides fringe positions to the realtime control system to enable fringe tracking. The Classic combiner produces three output beams, each including light from four of the six input beams; thus, each output beam has fringes from up to six baselines.

Our original intent was to disperse the light in each output beam into 32 spectral channels and to use all three output beams simultaneously, giving us 96 data streams in all. However, due to bandwidth limitations of the VME bus and the VxWorks ${ }^{(d)}$ real-time operating system, the backend data acquisition electronics were never fully implemented. As a result, we are limited to 32 data streams, which we have implemented as 16 spectral channels $(\lambda \lambda 550-850 \mathrm{~nm})$ in any two of the three output beams. This setup can sample as many as 11 baselines simultaneously, one of which is present on both output beams. The three output beams from the other side of the combiner are used in reverse to inject alignment beams and the whitelight source beam.

The limiting magnitude of the Classic combiner has improved, under the best seeing conditions, to $V=6^{\mathrm{m}} 7$ for a single baseline, or
$V=6^{\mathrm{m}} 0$ for multiple baselines with phase closure. The improvement is due in part to alignment refinements (e.g., explicitly optimizing the overlap of the stellar beams at the beginning of the night) and to changing from overcoated silver to moredurable bare aluminum for the siderostat and tiptilt mirrors, the only ones exposed to outdoor air.

Matthew Muterspaugh, Askari Ghasempour and colleagues at Tennessee State University (TSU) built the second beam combiner, the Visible Imaging System for Interferometric Observations at NPOI (VISION) (Ghasempour et al., 2012), with NSF funding. The design, very similar to that of the MIRC combiner (Monnier et al., 2004) at the CHARA array, uses single-mode polarizationpreserving fibers to spatially filter the incoming beams. The fibers are placed side by side and focused on a zero-read-noise EMCCD in an Andor Ixon DU-860 camera. (e) The spacing between fibers is nonredundant, producing up to 15 simultaneous distinct fringe patterns across the image. The image is dispersed vertically, with low resolution $(R=$ $200, \lambda \lambda 570-900 \mathrm{~nm})$ and high resolution $(R=$ $1200, \Delta \lambda=50 \mathrm{~nm}$ within the $\lambda \lambda 570-900 \mathrm{~nm}$ range) modes available. With some effort, a different set of single-mode fibers can be swapped for the normal VISION fibers, enabling operations at wavelengths near $\lambda 430 \mathrm{~nm}$. A separate camera monitors the light level in each input beam so fringe visibilities can be corrected for flux imbalance.

VISION's combination of improved detectors, better spatial filtering, photometric monitoring of the input beams, a more stable fringe modulation technique, and modern electronic and computer components is expected to lead to a gain of one magnitude in sensitivity and to significant improvement in how well these data can be calibrated.

\subsection{Control system}

The overall control system architecture remains as described in Armstrong et al. (1998b), with a highspeed network joining nodes at each siderostat and nodes for the fringe tracker, the FDLs, the sequence controller, and the observer's console. Nodes at the siderostats and the observer's console have been upgraded since 1998. The fringe tracker and FDL controller remain, but upgrades in progress are described below.

\footnotetext{
${ }^{\mathrm{e}}$ Andor Technology PLC, Belfast, UK; www.andor.com
}

${ }^{\mathrm{d} W i n d}$ River Systems, Inc., Alameda, CA; www.windriver. com 


\section{Operational Status}

The NPOI first combined six beams simultaneously in 2001, using the four astrometric stations as well as the imaging stations E02 and W07, $5 \mathrm{~m}$ and $56 \mathrm{~m}$ from the center of the array. In 2006, the siderostat at E02 was moved to E06, $35 \mathrm{~m}$ from the array center; since then, the operational status has remained largely unchanged, with all four astrometric siderostats available along with two imaging siderostats and baseline lengths ranging from $16 \mathrm{~m}$ to $79 \mathrm{~m}$ (Fig. 1). These lengths produce fringe spacings (a measure of angular resolution) ranging from 13 milliarcsec (mas) at $\lambda 850 \mathrm{~nm}$ on the $16 \mathrm{~m}$ baseline to 1.7 mas at $\lambda 550 \mathrm{~nm}$ on the $79 \mathrm{~m}$ baseline. However, one FDL delay cart is currently in use as a testbed for the new FDL controller, so a maximum of five of these stations can be used at a given time. When all six are available and in operation, the fact that the LDLs have not yet been integrated into the array limits sky coverage to a $2 \mathrm{hr}$ range in hour angle for declinations south of $\sim 70^{\circ}$.

We continue to use the Classic combiner for routine observations as the process of integrating the VISION combiner into the NPOI continues. Observations are carried out by a single observer, and are scheduled for all but about 10 nights per year.

One of the goals of the NPOI is to phase the array using multiple-baseline bootstrapping, in which each station in a chain of stations is phased to its nearest neighbors, so as to maximize the number of pixels across the image of a stellar surface (Armstrong et al., 1998a). However, the current fringe engine does not have the capacity to carry out those calculations fast enough to keep up with the atmosphere. Instead, one array element is chosen as the reference station, and group delay is tracked on the five baselines linking the reference to the remaining five stations. This constraint will be eliminated with the advent of a new fringe engine based on current technology.

\section{Progress}

Over the past four years, we have made significant progress toward a full buildout of the array, while collaborators at TSU and New Mexico Tech have received NSF grants that have resulted in hardware improvements. The following lists a variety of improvements, roughly in order of the stage of completion.
- Improvements to current siderostat stations. We have completed the installation of new domes, as well as improvements to enclosures for star acquisition and tip-tilt optics, at five stations.

- Control system upgrades. A second generation of controllers based on 80x86 computers running Linux CentOS 6.2 has been installed at the two current imaging siderostats, with the four astrometric stations scheduled for 2013, and with four more controllers for imaging stations to follow. We have upgraded the top-level control computer and software, and we are in the process of developing and testing upgrades to the "constant term" monitoring system and to the tip-tilt mirror control system.

- Classic combiner data acquisition. Anders Jorgensen (New Mexico Tech), Tim Buschmann (Arizona Embedded Systems LLC), and colleagues are implementing a field-programmable gate array (FPGA) based replacement for the current Classic data acquisition system that will sample the data in 128 bins per $2 \mathrm{~ms}$ and utilize all 32 spectral channels on all three output beams (Brown et al., 2012).

- Fringe engine. The computer that operates the new FPGA-based data acquisition system will replace the function of the current fringe engine and will be able to carry out the calculations needed for multi-baseline bootstrapping. The hardware is finished, and the firmware and software are under development. This system will be tested on the NPOI in the autumn of 2013.

- VISION. This second beam combiner for the NPOI is described above as well as by Ghasempour et al. (2012). The TSU team saw first fringes on one baseline in October 2012, and fringes on six baselines from four array elements in January 2013.

- Additional stations. With internal NRL funds, we have completed the infrastructure needed to place siderostats at stations E10 and W10, $250 \mathrm{~m}$ out along the east and west arms, and have partially completed the infrastructure at station N07, $56 \mathrm{~m}$ out along the north arm. We are now in the process of infrastructure completion at three close-in stations, E03, N03, and W04, at distances of $7.6 \mathrm{~m}, 8.6 \mathrm{~m}$, and $12.7 \mathrm{~m}$ from the center of the array (Fig. 2).

- FDL controllers. The NPOI uses laser metrology to measure the FDL positions. However, the electronics available 20 years ago were not fast 


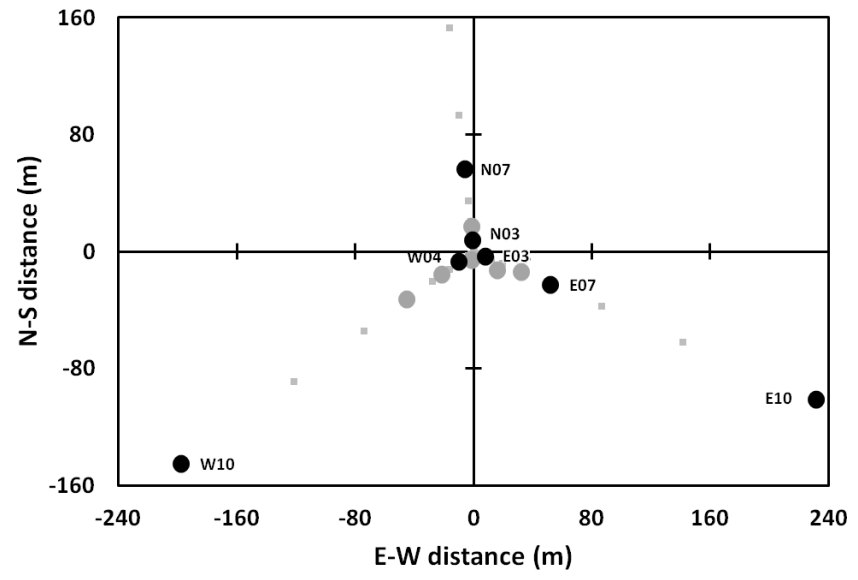

Fig. 2. Available stations after current upgrades. Filled black circles indicate the stations that are currently being added. Filled gray circles indicate the stations already available. As in Fig. 1, the small gray squares are station locations not yet in use. The north arm of the imaging array $\mathrm{Y}$ extends beyond the top of the figure.

enough to use those measurements for real-time adjustment of the $500 \mathrm{~Hz}$ triangle-wave delay modulation that the Classic combiner uses for fringe detection. An incorrect stroke shape leads to phase errors and loss of fringe contrast. With Arizona Embedded Systems, we are designing and building new FDL controllers capable of sampling the FDL positions 128 times per $500 \mathrm{~Hz}$ stroke. The fast sample rate and custom design will enable us to accurately calibrate the stroke waveform on a nightly basis if needed. The first of these new controllers is currently being tested with one of the FDL carts.

- LDL integration. Also with internal NRL funds, we have nearly completed the LDLs. We must still finish the development and installation of controllers for the pop-up mirrors, and we must fabricate modified mirror mounts for the periscopes to enable them to divert light to the LDLs before sending it on to the FDLs.

\section{Medium Term Improvements}

\section{1. $1.8 \mathrm{~m}$ telescopes}

In 2010, USNO accepted the four $1.8 \mathrm{~m}$ outrigger telescopes that were originally built for the Keck Interferometer, after NASA canceled their installation on Mauna Kea. Three of the telescopes are in storage in Tucson; the fourth is currently installed at Mt. Stromlo Observatory in Australia as part of a space debris tracking system (Smith, 2007). In 2012, we completed the infrastructure plans and drawings

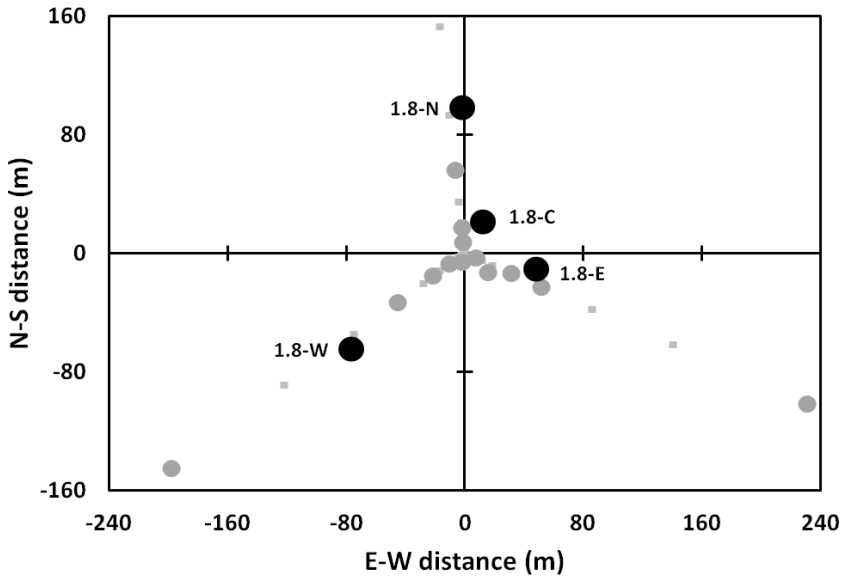

Fig. 3. Planned locations of the $1.8 \mathrm{~m}$ telescopes (filled black circles). Filled gray circles indicate the stations already available plus the new stations shown in Fig. 2. As in Figs. 1 and 2 , the small gray squares are station locations not yet in use.

and obtained the requisite special use permit from the US Forest Service. Our planned configuration is an irregular $\mathrm{Y}$ with baseline lengths from $46 \mathrm{~m}$ to $179 \mathrm{~m}$. Funding in fiscal years 2015-2019 is in place for the first stage of installation.

\section{2. $\quad 1.4 \mathrm{~m}$ telescopes}

In 2003, NRL began a program to develop lightweight $1.4 \mathrm{~m}$ telescopes and mounts made entirely from carbon fiber reinforced polymer (CFRP), with Composite Mirror Associates (CMA) of Tucson, Arizona (Restaino et al., 2008). This technology holds the promise of producing a telescope weighing a total of $\approx 120 \mathrm{~kg}$, well suited to reconfiguring the NPOI. The cost per telescope can be substantially lower than that of a conventional glass-primary telescope because the mandrel used to form the primary, which is the surface that must be figured and polished to optical precision, can be reused many times. In addition, the cost of the mount can be lower because of the telescope's reduced weight. At present, one prototype $1.4 \mathrm{~m}$ telescope is complete, and its mount is partially fabricated. We are seeking funding to fine-tune the figure of the mandrel, as well as to complete the mount.

\section{Observing Programs and Collaboration}

A primary observing interest of USNO at the NPOI is the NPOI Astrometric Catalog (UNAC) (Benson et al., 2010). Producing this catalog requires precise 
knowledge of the siderostat positions and of the internal optical pathlengths, all of which vary during the night with thermal changes. It also requires using the curvature of the fringe phases across the spectral channels to determine and remove the effects of atmospheric dispersion. This curvature is small in the infrared, but is stronger at visual wavelengths. We are currently reducing astrometric data for slightly over 100 stars.

All three members of the NPOI also pursue a variety of stellar astrophysics projects. A few recent examples include angular diameters of exoplanet host stars ( $\kappa \mathrm{CrB}$; Baines, Armstrong \& van Belle, 2013), orbits of Be star binaries ( $\delta$ Sco; Che et al., 2012; Tycner et al., 2011), dynamical masses of O stars ( $\zeta$ Ori A; Hummel et al., 2013), and binary orbits (Hummel, Zavala \& Sanborn, 2013) including Algol (Zavala et al., 2010). In addition, the NPOI was the first, and so far only, array to achieve an interferometric detection of a satellite (Hindsley et al., 2011).

Several of the astrophysical programs are led by outside observers. Particularly productive collaborations have focused on Be stars, particularly in the $\mathrm{H} \alpha$ line, (Schmitt et al., 2009; Koubský et al., 2010), Luminous Blue Variables (Balan et al., 2010), rapid rotators (e.g., Peterson et al., 2006), and on constraining stellar atmosphere models (e.g., Wittkowski et al., 2006). The NPOI is open to further collaborations, as well as to offering a limited amount of support to outside projects. Interested parties should contact one of the NRL, USNO, or Lowell authors.

\section{Conclusion}

When we began designing and building the NPOI, it was one of the world's most ambitious interferometer projects in terms of number of apertures, baseline length, and flexibility. Over the past two decades, interferometers with an equal number of larger apertures (CHARA) and with fewer but still larger apertures (VLTI, LBT Interferometer, Keck Interferometer) have been built and have become highly productive facilities. However, the NPOI was until recently the only operating interferometer at visual wavelengths, and is the only interferometer with wide-angle astrometric capabilities.

The developments now under way will allow us to reclaim the title for longest operating baseline. At least as important, they will allow us to start using the flexibility of configuration that is part of the original design, and to extend the number of links in a chain of bootstrapped baselines. This last capability is the key to observing the low fringe visibilities where the details in stellar surface imaging are to be found, not to mention its importance to NPOI's role as a testbed for satellite imaging. Finally, if we are able to find the support needed to complete the installation of the $1.8 \mathrm{~m}$ telescopes and to install the $1.4 \mathrm{~m}$ telescopes, the NPOI will be unmatched in its combination of aperture size, number of array elements, and range of baseline lengths.

\section{Acknowledgments}

The NPOI is a joint project of the Naval Research Laboratory and the US Naval Observatory in cooperation with Lowell Observatory, and is funded by the Oceanographer of the Navy and the Office of Naval Research. We thank the NSF for support for work at New Mexico Tech through grant AST-0909184 and for VISION at Tennessee State through grant AST-0958267, as well as the Lowell Observatory Predoctoral Fellowship program for continued support for work on VISION. MWM acknowledges support from Tennessee State University and from the State of Tennessee through its Centers of Excellence program.

\section{References}

Armstrong, J. T., Mozurkewich, D., Pauls, T. A. \& Hajian, A. R., 1998a, Bootstrapping the NPOI: Keeping long baselines in phase by tracking fringes on short baselines. In Reasenberg, R. D., ed., Astronomical Interferometry, Proc. SPIE, 3350, 461.

Armstrong, J. T., Mozurkewich, D., Rickard, L. J. et al., 1998b, The Navy Prototype Optical Interferometer (NPOI), ApJ, 496, 550.

Baines, E. K., Armstrong, J. T. \& van Belle, G. T., 2013, Navy Precision Optical Interferometer observations of the exoplanet host $\kappa$ Coronae Borealis and their implications for the star's and planet's masses and ages, ApJ, 771, L17.

Balan, A., Tycner, C., Zavala, R. T. et al., 2010, The spatially resolved $\mathrm{H} \alpha$-emitting wind structure of $\mathrm{P}$ Cygni, $A J, \mathbf{1 3 9}$, 2269 .

Benson, J. A., Hutter, D. J., Johnston, K. J. et al., 2004, NPOI: Recent technology and science. In Traub, W. A., ed., New Frontiers in Stellar Interferometry, Proc. SPIE, 5491, 1700.

Benson, J. A., Hutter, D. J., Zavala, R. T. et al., 2010, From fringes to the USNO Navy Prototype Optical Interferometer astrometric catalog. In Danchi, W. C., Delplancke, F. \& Rajagopal, J. K., eds., Optical and Infrared Interferometry II, Proc. SPIE, 7734, 77343K. 
Brown, M. F. D., Jorgensen, A. M., Buschmann, T., Hutter, D. J. \& Armstrong, J. T., 2012, A new, fast data acquisition system for the NPOI. In Delplancke, F., Rajagopal, J. K. \& Malbet, F., eds., Optical and Infrared Interferometry III, Proc. SPIE, 8445, 844530.

Che, X., Monnier, J. D., Tycner, C. et al., 2012, Imaging disk distortion of Be binary system $\delta$ Scorpii near periastron, ApJ, 757, 29.

Ghasempour, A., Muterspaugh, M. W., Hutter, D. J. et al., 2012, Building the next generation science camera for the Navy Optical Interferometer. In Delplancke, F., Rajagopal, J. K. \& Malbet, F., eds., Optical and Infrared Interferometry III, Proc. SPIE, 8445, 84450M.

Hindsley, R. B., Armstrong, J. T., Schmitt, H. R. et al., 2011, Navy Prototype Optical Interferometer observations of geosynchronous satellites, Appl. Optics, 50, 2692.

Hummel, C. A., Rivinius, Th., Nieva, M.-F. et al., 2013a, Dynamical mass of the O-type supergiant in $\zeta$ Orionis A, $A ध A, \mathbf{5 5 4}, 52$.

Hummel, C. A., Zavala, R. T. \& Sanborn, J., 2013b, Binary Studies with the Navy Precision Optical Interferometer, Cent. Eur. Astrophys. Bull., 37, 1, 127.

Hutter, D. J. \& Elias II, N. M., 2003, Array metrology system for an optical long-baseline interferometer. In Traub, W. A., ed., Interferometry for Optical Astronomy II, Proc. SPIE, 4838, 1234.

Hutter, D. J., Benson, J. A., Buschmann, T. et al., 2008, NPOI: Recent progress and future prospects. In Schöller, M., Danchi, W. C. \& Delplancke, F., eds., Optical and Infrared Interferometry, Proc. SPIE, 7013, 701306.

Johnston, K. J., Benson, J. A., Hutter, D. J. et al., 2006, The Navy Prototype Optical Interferometer: Recent developments since 2004. In Monnier, J. D., Schöller, M. \& Danchi, W. C., eds., Advances in Stellar Interferometry, Proc. SPIE, 6268, 626806.

Koubský, P., Hummel, C. A., Harmanec, P. et al., 2010, Properties and nature of Be stars, $A \boxminus A, \mathbf{5 1 7}, \mathrm{A} 24$.

Monnier, J. D., Berger, J.-P., Millan-Gabet, R. \& ten Brummelaar, T., 2004, The Michigan Infrared Combiner
(MIRC): IR imaging with the CHARA array. In Traub, W. A., ed., New Frontiers in Stellar Interferometry, Proc. SPIE, 5491, 1370.

Mozurkewich, D., 1994, Hybrid design for a six-way beam combiner. In Breckinridge, J. B., ed., Amplitude and Intensity Spatial Interferometry II, Proc. SPIE, 2200, 76.

Mozurkewich, D., Benson, J. A. \& Hutter, D. J., 2003, NPOI: A progress report. In Traub, W. A., ed., Interferometry for Optical Astronomy II, Proc. SPIE, 4838, 53.

Peterson, D. M., Hummel, C. A., Pauls, T. A. et al., 2006, Vega is a rapidly rotating star, Nature, 440, 13, 896.

Restaino, S. R., Martinez, T., Andrews, J. R. et al., 2008, Meter class Carbon Fiber Reinforced Polymer (CFRP) telescope program at the Naval Research Laboratory. In Atad-Ettedgui, E. \& Lemke, D., eds., Advanced Optical and Mechanical Technologies in Telescopes and Instrumentation, Proc. SPIE, 7018, 70183C.

Schmitt, H. R., Pauls, T. A., Tycner, C. et al., 2009, Navy Prototype Optical Interferometer imaging of line emission regions of $\beta$ Lyrae using differential phase referencing, ApJ, 691, 984.

Shao, M., Colavita, M. M., Hines, B. E. et al., 1988, The Mark III stellar interferometer, $A \mathscr{E} A, \mathbf{1 9 3}, 357$.

Smith, C. H., 2007, The EOS space debris tracking system. In Proc. 2006 AMOS Conference, Kihei, HI, p. 719 (www.amostech.com/TechnicalPapers/2006/ Satellite_Metrics/Smith.pdf).

Tycner, C., Ames, A., Zavala, R. T. et al., 2011, The revised orbit of the $\delta$ Sco system, ApJ Lett., 729, L5.

Wittkowski, M., Hummel, C. A., Aufdenberg, J. P. \& Roccatagliata, V., 2006, Tests of stellar model atmospheres by optical interferometry III. NPOI and VINCI interferometry of the M0 giant $\gamma$ Sagittae covering $0.5-2.2 \mu \mathrm{m}$, $A \mathscr{G} A, \mathbf{4 6 0}, 843$.

Zavala, R. T., Hummel, C. A., Boboltz, D. A. et al., 2010, The Algol triple system spatially resolved at optical wavelengths, ApJ, 715, 44. 